\title{
"Bilateral Coronoidotomy in Conjunction With Submucous Fibrous Bands Release As A Treatment Modality for Oral Submucous Fibrosis"
}

\author{
Dr. Ayesha ${ }^{1}$,Dr. Veeresh $\mathrm{M}^{2}$ \\ ${ }^{1}$ kmio Bangalore. \\ ${ }^{2}$ professor And Head Dept. Of Dentistry Esicmc \& Pgimsr Bangalore
}

\begin{abstract}
Purpose: Oral submucous fibrosis (OSMF) is a premalignant condition mainly associated with chewing areca nut. It is characterized by inflammation, increased deposition of submucosal collagen and fibrotic bands in the oral tissues, which increasingly limit mouth opening. Thus causing difficulty in mastication, speech, swallowing, maintaining oral hygiene and decreases patients' quality of life.

We evaluated efficacy of coronoidotomy at improving patient's mouth opening.

Aim And Objective: To evaluate the efficacy of coronoidotomy along with fibrotic bands release as surgical intervention in advanced oral submucous fibrosis.

Method: Ten patients diagnosed as Group III and IV OSMF (Khanna-Andrade Classification 1995) were included in the study. Preoperative clinical findings and interincisal distance were assessed. All patients underwent bilateral coronoidotomy along with release of fibrous bands and grafting of defect with bovine collagen membrane with 3 months follow up. The interincisal distance is recorded at time intervals of $1^{\text {st }}$ day, $7^{\text {th }}$ day, $I^{\text {st }}$ month and $3^{\text {rd }}$ months postoperatively.

Results: Intraoperative interincisal mouth opening of about a mean of $28 \mathrm{~mm}$ obtained after fibrotic bands release. Further, after bilateral coronoidotomy, a mean Intraoperative mouth opening of $42 \mathrm{~mm}$ was achieved. On first postoperative day a mean mouth opening of $38.3 \mathrm{~mm}$ was observed. Regular mouth opening exercises commenced on the third postoperative day. Both the frequency and duration was increased which aided in further increasing the mouth opening. Mean interincisal distance of $37.1 \mathrm{~mm}$ was achieved after 7 days, 34.6.mm at first month follow up and $32.9 \mathrm{~mm}$ after 3 months.

Conclusion: The procedure bilateral coronoidotomy with the resection of fibrous bands was effective in management in Group III and IV OSMF for long term results.
\end{abstract}

Keywords: Oral Submucous Fibrosis, interincisal distance, Bilateral Coronoidotomy.

\section{Introduction}

Oral Submucous fibrosis is an insidious, chronic disease affecting any part of the oral cavity \& sometimes the pharynx. Although occasionally preceded by and/or associated with vesicle formation, it is always associated with juxtaepithelial inflammatory reaction, followed by a fibroelastic change of the lamina propria with epithelial atrophy, leading to stiffness of the oral mucosa and causing trismus and inability to eat. (Pindborg \& Sirsat 1966) ${ }^{1}$.

This condition was first described by Schwartz in 1952, which he called "atrophia idiopathica (tropica) mucosae oris". Joshi (1953) is credited to be the first person who described this condition and gave it the present term. The other names are "diffuse oral Submucous fibrosis", "idiopathic scleroderma of the mouth", "idiopathic palatal fibrosis", "sclerosing stomatitis" and "juxta-epithelial fibrosis.

The various hypotheses put forward so far suggest a multifactorial origin for this condition. Alongside the role of local irritants such as capsaicin, tobacco, areca nut, pungent and spicy foods, and alcohol, an underlying systemic predisposition is likely because of the geographical and ethnic distribution of OSMF. Among the systemic factors, the main ones incriminated are chronic iron and vitamin B-complex deficiency, anaemia, and a genetic predisposition to the disease. ${ }^{2}$

The symptoms and signs of OSMF are due to inflammation and primarily fibrosis. The most common initial symptoms and signs are a burning sensation. In the more advanced stage, the essential feature is fibrous bands restricting mouth opening. The most morbid clinical symptom being the restriction of mouth opening for which the patient reaches out for treatment. The inability to open the mouth jeopardizes the quality of life through impairing eating, interfering with oral hygiene, restricting access for dental procedures, and affecting speech and facial appearance.

Treatment of OSMF is a challenge as the disease progresses. Conservative treatment for initial stages of OSMF includes vitamins, iron supplements, intralesional injections of hyaluronidase, placental extracts and 
steroids $^{3}$. Submucosal injections of various drugs may produce temporary symptomatic relief ${ }^{4}$ but can lead to aggravated fibrosis, pronounced trismus and increased morbidity, from the mechanical injury secondary to insertion of the needle and chemical irritation from the $\mathrm{drug}^{3,5}$.

Various surgical procedures have been proposed to address trismus by different authors with variable success rates. The mainstay in the treatment of submucous fibrosis is concentrated on attempts to improve the mouth opening and relieve the symptoms by medicinal or surgical means. This includes excision of fibrous bands and reconstruction of defects. Simple release of fibrosis showed recurrence due to scarring. Thus, additional procedures, such as splitting the temporalis tendon, coronoidectomy and masseter muscle stripping ${ }^{6,7}$ have also been described to enhance mouth opening.

\section{Patients And Methods}

Ten patients ( 8 men and 2 women aged between 25 and 31years) who reported with chief complaint of long standing difficulty in mouth opening and positive history of betel nut, supari, tobacco chewing, with or without lime. All patients had interincisal mouth opening of less than $26 \mathrm{~mm}$. Patients were thoroughly examined for symptoms like burning mouth sensation, decrease salivation, inability to blow and pain in the ears and clinically palpable fibrotic bands running across the buccal mucosa, any blanching in the retromolar area, anterior and posterior faucial pillars, hard and soft palate and uvula was noted. The biopsy of the buccal mucosa done to histopathologically confirm as Oral Submucous Fibrosis of Group III and Group IV (Khanna \& Andrade classification, 1995) were included in this study.

The procedure is carried out under general anaesthesia given through nasoendotracheal intubation. Preoperative interincisal opening is measured (Fig.1). The surgical technique involves release of fibrotic bands using electrosurgical knife. The incision is placed in the buccal mucosa along the occlusal line starting from the angle of the mouth extending posteriorly upto the retromolar region, bilaterally to the depth of submucosal layer.All fibrotic tissues under the buccal mucosa are released bilaterally (Fig.2). The forced interincisal mouth opening is noted after fibrous bands release.

Further, incision is placed along the anterior border of the ramus and subperiosteal dissection is done to reach up to sigmoid notch wherein isolation of the coronoid process. The coronoidotomy (Fig.3) is performed using straight fissure bur. Similarly done on the other side. Then, the interincisal distance noted after coronoidotomy (Fig.4). A mean mouth opening of 35-40mm was considered acceptable on operation table. The intraoral defect is covered using bovine collagen membrane which was secured along the periphery and quilting sutures were done to attain close apposition using absorbable suture. Postoperatively, intensive jaw physiotherapy advised. The patients were regularly supervised and evaluated for mouth opening at intervals of $1^{\text {st }}$ day, $7^{\text {th }}$ day, $1^{\text {st }}$ month (Fig.5) and $3^{\text {rd }}$ month (Fig.6) postoperatively.

\section{Results}

The patients included in the study were in the age group of 25-37 years comprising of 2 females and 8 males.

The preoperative spontaneous interincisal distance was in the range of 1-26 mm. After release of fibrotic bands, the Intraoperative interincisal mouth opening of about a mean of $28 \mathrm{~mm}$ obtained. Further, bilateral coronoidotomy was done and a mean intraoperative mouth opening of $42 \mathrm{~mm}$ was achieved. On the first postoperative day a mean mouth opening of $38.3 \mathrm{~mm}$ was observed. Regular mouth opening exercises commenced on the third postoperative day. Both the frequency and duration was increased which aided in further increasing the mouth opening. Mean interincisal distance of $37.1 \mathrm{~mm}$ was achieved after 7 days, 34.6. $\mathrm{mm}$ at first month follow up and $32.9 \mathrm{~mm}$ after 3 months. The patients were motivated both pre and post operative for the cessation of habit and mouth opening exercises. The details of the postoperative mouth opening evaluation are tabulated in Table.1. The mean values of postoperative interincisal mouth opening in comparison with preoperative measurement is shown in Graph.1.

Table 1: Mouth Opening (mm) in Oral Submucous Fibrosis Cases

\begin{tabular}{|c|c|c|c|c|c|c|c|c|c|}
\hline \multicolumn{3}{|c|}{ Patients } & \multicolumn{7}{|c|}{ INTERINCISAL MOUTH OPENING (Mm) } \\
\hline \multirow[b]{2}{*}{$\begin{array}{l}\text { Sl. } \\
\text { No. }\end{array}$} & \multirow[b]{2}{*}{$\begin{array}{l}\text { Age } \\
\text { Yrs }\end{array}$} & \multirow[b]{2}{*}{ Sex } & \multirow[b]{2}{*}{$\begin{array}{l}\text { Preop } \\
\text { (Mm) }\end{array}$} & \multicolumn{2}{|c|}{ Intraoperatively } & \multicolumn{4}{|c|}{ Postoperatively } \\
\hline & & & & $\begin{array}{l}\text { After Fibrotic } \\
\text { Bands } \\
\text { Release(Mm) } \\
\end{array}$ & $\begin{array}{l}\text { After } \\
\text { Coronoidotom } \\
\text { y }(\mathrm{Mm})\end{array}$ & $\begin{array}{l}1^{\text {st }} \\
\text { Day }\end{array}$ & $7^{\text {th }}$ Day & $\begin{array}{l}1^{\text {st }} \\
\text { Mnth }\end{array}$ & $\begin{array}{l}3^{\text {rd }} \\
\text { Mnth }\end{array}$ \\
\hline 1 & 29 & $\mathbf{F}$ & 7 & 20 & 45 & 32 & 30 & 25 & 25 \\
\hline 2 & 30 & M & 1 & 25 & 40 & 36 & 34 & 30 & 28 \\
\hline 3 & 37 & M & 10 & 30 & 40 & 37 & 37 & 35 & 35 \\
\hline 4 & 25 & $\mathbf{M}$ & 20 & 30 & 40 & 38 & 37 & 35 & 35 \\
\hline 5 & 29 & M & 24 & 30 & 40 & 39 & 37 & 35 & 33 \\
\hline
\end{tabular}


“Bilateral Coronoidotomy In Conjunction With Submucous Fibrous Bands....

\begin{tabular}{|l|l|l|l|l|l|l|l|l|l|}
\hline $\mathbf{6}$ & $\mathbf{2 6}$ & M & $\mathbf{2 3}$ & $\mathbf{3 5}$ & $\mathbf{4 5}$ & $\mathbf{4 2}$ & $\mathbf{4 2}$ & $\mathbf{4 0}$ & $\mathbf{4 0}$ \\
\hline $\mathbf{7}$ & $\mathbf{2 7}$ & M & $\mathbf{2 6}$ & $\mathbf{3 0}$ & $\mathbf{4 5}$ & $\mathbf{4 3}$ & $\mathbf{4 0}$ & $\mathbf{3 8}$ & $\mathbf{3 8}$ \\
\hline $\mathbf{8}$ & $\mathbf{3 1}$ & F & $\mathbf{7}$ & $\mathbf{2 7}$ & $\mathbf{4 0}$ & $\mathbf{3 8}$ & $\mathbf{3 8}$ & $\mathbf{3 5}$ & $\mathbf{3 0}$ \\
\hline $\mathbf{9}$ & $\mathbf{2 8}$ & M & $\mathbf{2 5}$ & $\mathbf{3 0}$ & $\mathbf{4 5}$ & $\mathbf{4 0}$ & $\mathbf{3 8}$ & $\mathbf{3 8}$ & $\mathbf{3 5}$ \\
\hline $\mathbf{1 0}$ & $\mathbf{3 1}$ & M & $\mathbf{7}$ & $\mathbf{2 7}$ & $\mathbf{4 0}$ & $\mathbf{3 8}$ & $\mathbf{3 8}$ & $\mathbf{3 5}$ & $\mathbf{3 0}$ \\
\hline
\end{tabular}

Graph 1: Depicting the mean preoperative, Intraoperative after fibrotomy and coronoidotomy, postoperative 1 day, postoperative $7^{\text {th }}$ day, postoperative 1 month and postoperative 3 months interincisal distance.

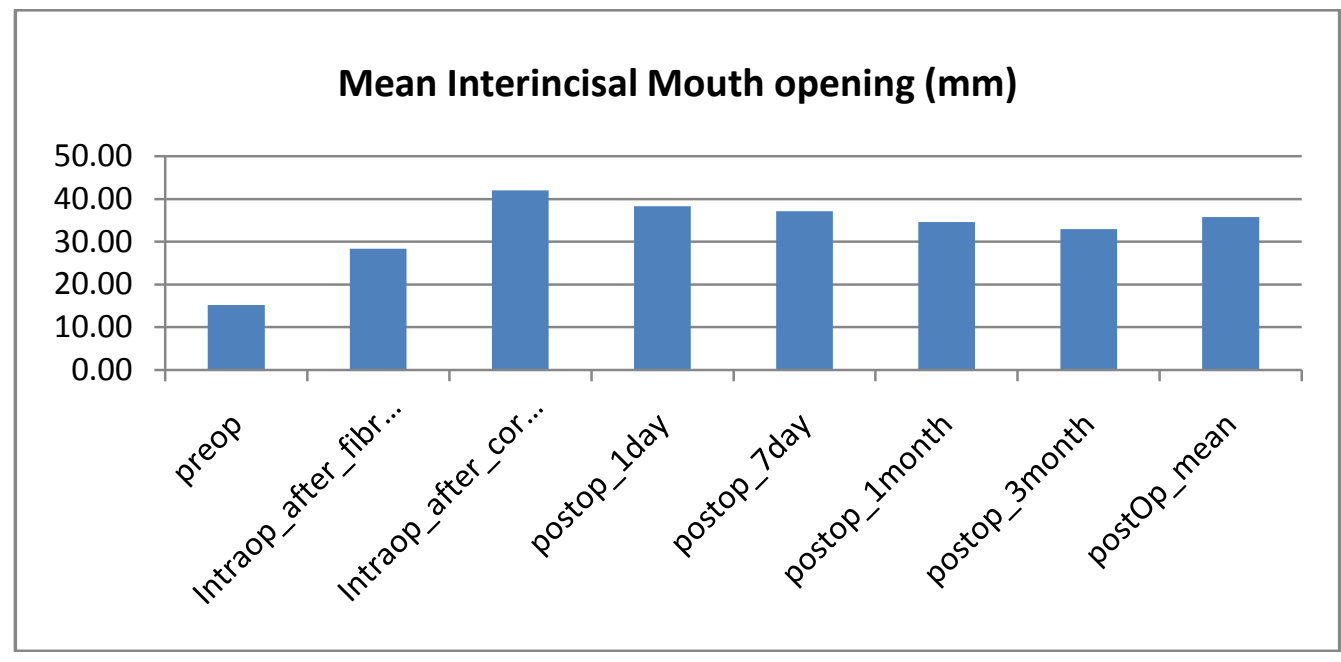

\section{Discussion}

Oral Submucous fibrosis (OSMF) is a premalignant condition mainly associated with the practice of chewing betel quid containing areca nut. The prevalence rate in India is about $0.2-0.5 \%$ and prevalence by gender varying from $0.2 \%$ to $2.3 \%$ in males and $1.2 \%$ to $4.57 \%$ in females. ${ }^{8}$ It is predominantly a disease of oral cavity and oropharynx. It is slowly progressive crippling disease in which fibrous bands form in juxtaepithelial layer of oral mucosa leading to severe restriction of mouth opening. The etiology still remains obscure and common irritants like betel nut, chillies, tobacco etc., are thought to be the underlying cause in the pathogenesis of OSMF. ${ }^{9}$

Pillai et $\mathrm{al}^{10}$ also concluded in his review that the etiology is unknown but is probably multifactorial. Main contributing factor as thought by Jayanthi et $\mathrm{al}^{11}$, is the use of pan which typically consists of areca nut, tobacco and crude lime wrapped in betel leaf. The onset of this condition is insidious, majority of patients with OSMF present with prodromal symptoms such as burning sensation in the mouth when consuming spicy food, appearance of blisters especially in the palate.The hallmark of the disease is submucosal fibrosis that affects most parts of the oral cavity, pharynx and upper third of the oesophagus. ${ }^{12}$ As the disease progresses, the oral mucosa becomes blanched and slightly opaque, and white fibrous bands appear. ${ }^{10}$ In late stages, rigidity of lip, tongue and palate leads to varying degrees of limitation of mouth opening and tongue movement due to severe scarring which causes trismus.

OSMF has a high rate of morbidity because it causes a progressive inability to open the mouth, resulting in difficulty in eating and consequent nutritional deficiencies. It also has a significant mortality rate because it can transform into oral cancer, particularly squamous cell carcinoma, with reported incidence rate of $3 \%-7.6 \%{ }^{13}$.

Owing to the obscure etiology of the disease and morbidity, the mainstay of the treatment is concentrated on attempts to improve mouth opening and relieve the symptoms by medicinal or surgical means .The treatment of Oral Submucous fibrosis is based on severity of disease. Typically, if the disease is noted before development of trismus, cessation of the betel habit will often resolve the disease ${ }^{15,16}$. Once trismus has developed and disease is considered mild to moderate, the treatment at this stage is focused on restoring mandibular range of motion ${ }^{16}$.

Non-surgical treatments have so far yielded inconsistent results. Surgical treatment of severe trismus in submucous fibrosis patients can improve articulation, mastication, and oral hygiene, which are socially and functionally important. Thus, surgical treatment is the method of choice in patients with marked limitation of mouth opening, but has been reported to give rise to varying results.Multiple surgical modalities have been attempted, from moderately invasive to significantly invasive ${ }^{16}$. The various surgical procedures include excision 
of fibrous bands and reconstruction of defect with grafts. Release of fibrous bands can be done with electrosurgical blade ${ }^{17}$ or using laser.

Simple release of fibrosis has showed recurrence due to scarring and graft contraction. For cases in which initial surgical intervention is unsuccessful, more aggressive surgical therapy is indicated ${ }^{1,5,13,18,19}$. Often in this situation, a larger soft tissue buccal defect is created, needing large soft tissue reconstruction.

Additional procedures like splitting of temporalis tendon, coronoidectomy and masseter muscle stripping ${ }^{20}$ have also been described to enhance mouth opening. The importance of coronoidectomy and masticatory muscle myotomy and postoperative rehabilitation was also emphasized by Canniff and Harvey (1986), Khanna and Andrade ${ }^{14}$ (1995) followed by Yang-Ming Chang ${ }^{4}$ (2004) in the prevention of relapse due to postoperative inactivity and scarring ${ }^{4,14}$.The mean pre-operative interincisal distance (ID) in all ten OSMF cases of our study were $15.2 \mathrm{~mm}$ as compared to 7-24 mm (mean: $15.5 \mathrm{~mm}$ ) in study by Yang-Ming Chang et al. and $14.7 \mathrm{~mm}$ in study by M.C. Kothari et al.

M.C. Kothari et al (2012) evaluated ten patients with advanced (Stage III-IV) oral submucous fibrosis who underwent coronoidectomy, masticatory myotomy followed by vigorous mouth opening exercises. Results showed a mean interincisal opening of $14.7 \mathrm{~mm}$ preoperatively and $32.5 \mathrm{~mm}$ at 12 months postoperatively. In our study, on the first postoperative day a mean mouth opening of $38.3 \mathrm{~mm}$ was observed. Regular mouth opening exercises commenced on the third postoperative day. Both the frequency and duration was increased which aided in further increasing the mouth opening. Mean interincisal distance of $37.1 \mathrm{~mm}$ was achieved after 7 days, 34.6. $\mathrm{mm}$ at first month follow up and 32.9mm after 3 months. Results suggest this regime is effective.

The treatment of severe trismus requires a combination of surgical intervention and postoperative rehabilitation; the latter is essential for preventing a relapse due to postoperative inactivity and scarring. ${ }^{15}$

\section{Conclusion}

The initial stages on oral submucous fibrosis can be managed by conservative treatment and simple release of fibrous bands but severe degree of trismus as seen in advanced fibrosis has to be addressed surgically requiring release of fibrous bands and coronoidotomy to obtain adequate mouth opening.

In this study, surgical treatment was considered to be the solution in group III and group IV cases, with fibrous bands release and bilateral coronoidotomy as a highly effective surgical procedure in providing good long term results.

\section{References}

[1]. Pindborg J.J, Sirsat S.M; Oral Submucous Fibrosis, Oral Surg 1966; 22(6): 764-79.

[2]. Rajendran.OSMF: Etiology, pathogenesis and future research. Bulletin of WHO 1994, 72(6): 985-996.

[3]. Xiaowen Jiang, Jing Hu. Drug treatment of oral sub-mucous fibrosis: a review of the literature. J Oral Maxillofac Surg 2009;67:1510-5.

[4]. Yang-Ming Chang, Chi-Ying Tsai, Morten Kildal, and Fu-Chan Wei: Importance of Coronoidotomy and Masticatory Muscle Myotomy in Surgical Release of Trismus Caused by Submucous Fibrosis: Plastic and Reconstructive Surgery, June 2004; 113(7): 1940-1948.

[5]. Mehrotra D, Pradhan R, Gupta S. Retrospective comparison of surgical treatment modalities in 100 patients with oral submucous fibrosis. Oral Surg Oral Med Oral Pathol Oral Radiol Endod 2009;107:e1-0.

[6]. M. C. Kothari, N. Hallur, B. Sikkerimath, S. Gudi, C. R. Kothari. Coronoidectomy, masticatory myotomy and buccal fat pad graft in management of advanced oral submucous fibrosis. Int. J. Oral Maxillofac. Surg. 2012; 41(11): 1416-21.

[7]. Jens J. Pindborg, Hemming E. Poulsenm, Jacob Zachariah. Oral epithelial changes in thirty Indians with oral cancer and oral submucous fibrosis. Cancer 1967; 20: 1141-46.

[8]. Joseph AP, Rajendran R. Submucosa precedes lamina propria in initiating

[9]. fibrosis in oral submucous fibrosis - evidence based on collagen histochemistry. J Oral Maxillofac Pathol 2010; 1(1) ISSN 0976-1225.

[10]. Chandramani B. More, Sunanda Das, Hetul Patel, Chhaya Adalja, Vaishnavee Kamatchi, Rashmi Venkatesh. Proposed clinical classification for oral submucous fibrosis. 2012; 48: 200-202.

[11]. Pillai R, Balaram P, Reddiar KS. Pathogenesis of oral submucous fi brosis. Relationship to risk factors associated with oral cancer. Cancer, 1992, 69: 2011-2020.

[12]. Jayanthi V, Probert CSJ, Sher KS, Mayberry JF. Oral submucosal fi brosis - a preventable disease. Gut, 1992, 33: 4-6.

[13]. W.M. Tilakaratne, M.F. Klinikowski, Takashi Saku ,T.J. Peters, Saman Warnakulasuriya. Oral submucous fibrosis: Review on aetiology and pathogenesis. Oral Oncology (2006) 42, 561- 568.

[14]. Khanna JN, Andrade NN. Oral submucous fibrosis: a new concept in surgical manage-ment. Report of 100 cases. Int J Oral Maxillofac Surg 1995; 24:433-9.

[15]. C.Yeh. Application of the buccal fat pad to the surgical treatment of oral submucous fibrosis. Int. J. Oral Maxillofac. Surg. 1996; 25: $130-133$.

[16]. Abhinav Kumar, Anjana Bagewadi, Vaishali Keluskar, and Mohitpal Singh. Efficacy of Lycopene in the management of oral submucous fibrosis. OOOE 2007; 103 (2): 207-213.

[17]. Shahid R. Aziz: Oral Submucous Fibrosis: Case Report and Review of Diagnosis and Treatment: J Oral Maxillofac Surg 2008; 66:2386-2389.

[18]. Lai DR, Chen HR, Lin LM, Huang YL, Tsai CC. Clinical evaluation of different treatment methods for oral submucous fibrosis. A 10-year experience with 150 cases. J Oral Pathol Med 1995; 24:402-6. 
[19]. Ranganathan K, Gauri Mishra. Overview of classification schemes for oral submucous fibrosis. Journal of oral and maxilla Facial Pathology. 2006; 10(2):55-58.

[20]. S. M. Haider, A. T. Merchant, F. F. Fikree, M. H. Rahbar. Clinical and functional staging of oral submucous fibrosis. Br J of Maxillo Surg 2000; 38: 12-15.

[21]. Borle RM, Borle SM. Management of oral submucous fibrosis: a conservative approach. J Oral Maxollofac Surg 1991; 49:78891.
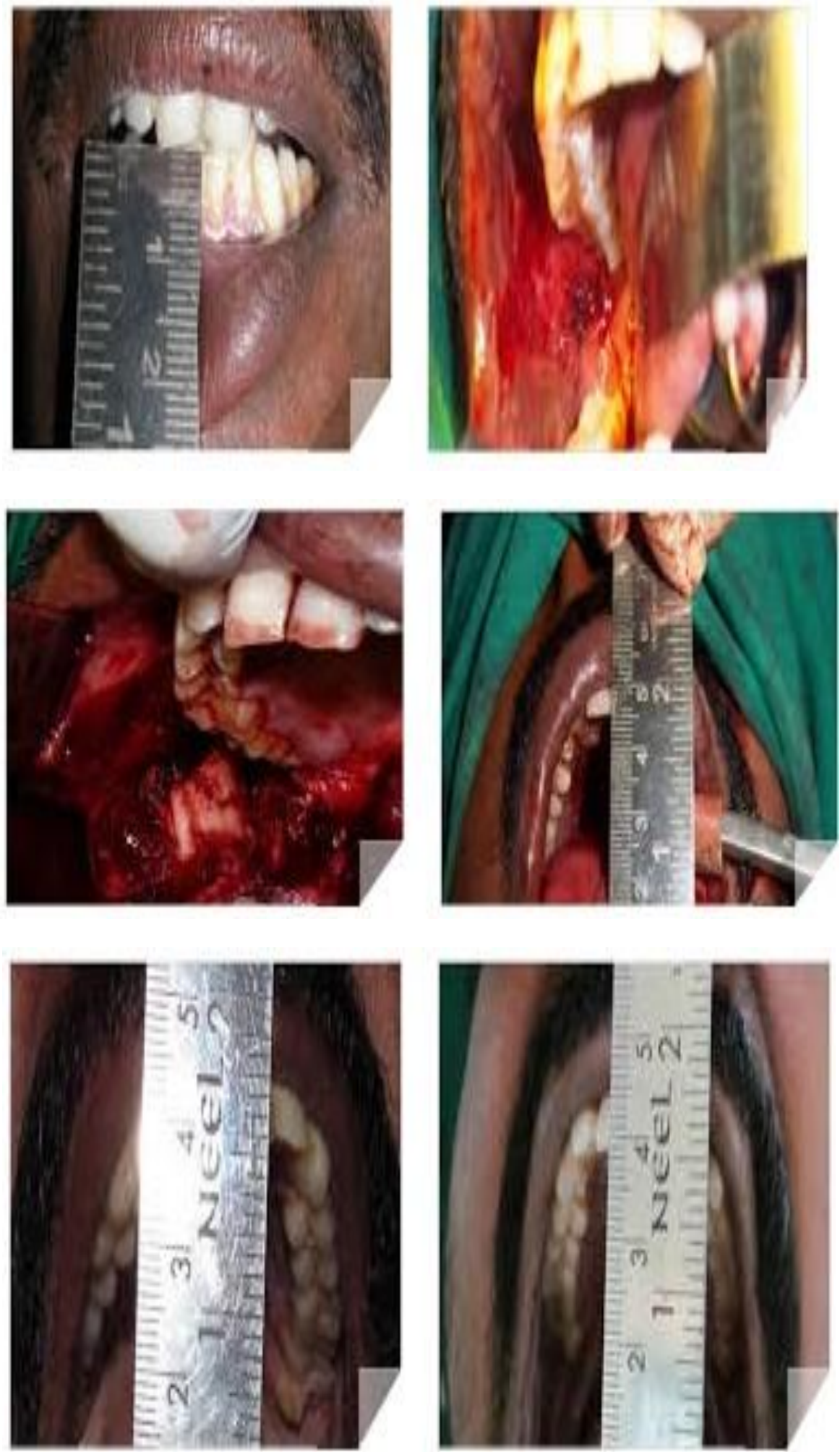